\title{
T7-like bacteriophage promoters: stress-induced duplex destabilization suggests a role in replication
}

\author{
M. Orlov*, A.Ryasik, E. Zykova, A. Sorokin \\ Institute of Cell Biophysics SB RAS, Pushchino, Russia \\ *e-mail: orlovmikhailanat@gmail.com
}

Key words: bacteriophage T7, SIDD, DNA physics, genomics, replication

Motivation and Aim: The fact that replication and transcription are coupled in prokaryotes has been known for many years, but a solid mechanistic explanation for it is lacking. T7-like bacteriophages are well-suited model systems to study the phenomenon since their small genomes contain both multiple promoters (being recognized by two different RNA-polymerases) and replication origins. Moreover, appreciable fraction of the promoter regions is known to be involved in transcription initiation [1].

Methods and Algorithms: DNA-protein interactions that govern various regulatory events are due to physicochemical properties of the molecules. Among them, one of the most important is stress-induced DNA duplex destabilization (SIDD). Sites predicted to be susceptible to duplex destabilization are found to be significantly associated with regulatory regions. SIDD profiles are calculated by means of statistical mechanics method evaluating the equilibrium distribution among states of denaturation of a DNA molecule under a defined level of superhelicity imposed [2]. Here we report analysis of genome-wide SIDD profiles for T7-like bacteriophages with the respect to regulatory site locations.

Results: Among T7-DNA native promoters ones functioning as secondary replication origins were found to be highly destabilized. The latter includes phiOL and phiOR located at both termini of several T7-like bacteriophage DNA (including T7). In all cases the two promoters were shown to coincide with highly destabilized duplex regions. For comparison purposes, selected T7-like genomes that lack phiOL and phiOR were additionally considered. In some cases sharp SIDD profile maxima were found to be located near one or both DNA termini, which implies presence of promoters analogous to phiOL and phiOR. Similar promoters might also be present in other bacteriophages.

Conclusion: The connection between promoter SIDD as a DNA duplex feature and specific involvement in replication has been evidenced for T7-like bacteriophage promoters involved in initiation of replication. This provides additional insight into mechanisms underlying link between transcription and replication and highlights importance of DNA duplex stability.

\section{References}

1. Wang H.Q., Benham C.J. (2006) Promoter prediction and annotation of microbial genomes based on DNA sequence and structural responses to superhelical stress. BMC Bioinformatics. 7:248-262.

2. Dunn J.J., Studier F.W., Gottesman M. (1983) Complete nucleotide sequence of bacteriophage T7 DNA and the locations of T7 genetic elements. J. Mol. Biol. 166:477-535. 